\title{
Voltage Stability Analysis and Bifurcation Techniques: A Detailed Survey
}

\author{
BV Srikanth \\ Deputy Manager, Electrical Divison, \\ SHAR, Sriharikota \\ Dr A Lakshmi Devi \\ Professor \& HOD, Dept of EEE \\ SVU College of Engineering, Tirupati
}

\begin{abstract}
Power systems are affected by events that depend upon the state of the power system. Voltage instability is caused due to heavily operated transmission lines and insufficient availability of reactive power. Main causes for the voltage instability are increased loading of transmission lines, Reactive power constraints, Load characteristic at low voltages, Response of On Load Tap Changing (OLTC) transformer, Unexpected relay operations etc. Some of the methods analyzing Voltage Stability are Optimal Power Flow, Continuation Power Flow, Voltage Stability Indices Calculation Method, Bifurcation Analysis and finding Chaotic Conditions. A detailed survey on Voltage Stability and different techniques available in the literature are illustrated. In this paper, Toolboxes available in the literature for analyzing Voltage Stability are explained.
\end{abstract}

Keywords: Voltage Stability, Voltage Instability, Voltage Collapse, PV Curves, Saddle Node Point and Chaotic Theory.

\section{INTRODUCTION}

In today's scenario, as the development is taking place simultaneously the demand for the electricity in the world is also increasing. Operation and planning of large interconnected power systems are becoming more and more complex. In power system, voltage stability plays a very important role. Voltage instability may cause blackouts and collapse of the power system. In last decade, many countries have experienced the problem of blackouts due to voltage instability. The problem of voltage stability is mainly caused due to stressed loading condition of the system [1]. It is essential to analyze the voltage stability for improvement in the efficiency of power system. Various authors have conducted research on analysis of voltage stability and methods to improve it. This paper, presents a review on research and development in the voltage stability analysis and methods for improving the voltage stability.

Power systems are affected by events that depend upon the state of the power system [1]. For instance, as loads increase, the flows on transmission lines increase. When the flow on a line exceeds a certain limit for a certain time period, a relay will open a circuit breaker removing the line from the network. The operation of the relay is triggered by the state of the transmission line (voltage, current, temperature, power), and the state is determined by system parameters such as load and import levels. The opening of the circuit breaker and removal of the line from the network in turn causes the flows on other lines to change and can lead to cascading events and even the loss of system stability.

\section{VOLTAGE STABILITY AND COLLAPSE}

According to the IEEE, the voltage stability is defined as the capacity of a power system to maintain in all nodes acceptable voltage levels under normal conditions after a system disturbance for a given initial condition [2]. This definition gives us an idea of the robustness of a power system which is measured by its capability of keep the equilibrium between the demanded load and the generated power.

Voltage collapse usually occurs on power system which is heavily loaded or faulted or has shortage of reactive power. Voltage collapse is a system instability involving many power system components [2]. In fact, a voltage collapse may involve an entire power system. Voltage collapse is typically associated with reactive power demand 
of load not being met due to shortage in reactive power production and transmission. Voltage collapse is a manifestation of voltage instability in the system.

Voltage stability is concerned with the ability of a power system to maintain steady voltages at all buses in the system under normal operating conditions, and after being subjected to a disturbance. Instability that may result occurs in the form of a progressive fall or rise of voltage of some buses. The possible outcome of voltage instability is loss of load in the area where voltages reach unacceptably low values, or a loss of integrity of the power system. Progressive drop in bus voltages can also be associated with rotor angles going out of step. For example, the gradual loss of synchronism of machines as rotor angles between two groups of machines approach or exceed 1808 would result in very low voltages at intermediate points in the network close to the electrical center [1]. In contrast, the type of sustained fall of voltage that is related to voltage instability occurs where rotor angle stability is not an issue. The main factor contributing to voltage instability is usually the voltage drop that occurs when active and reactive power flow through inductive reactance associated with the transmission network; this limits the capability of transmission network for power transfer. The power transfer limit is further limited when some of the generators hit their reactive power capability limits. The driving forces for voltage instability are the loads; in response to a disturbance, power consumed by the loads tends to be restored by the action of distribution voltage regulators, tap changing transformers, and thermostats. Restored loads increase the stress on the high voltage network causing more voltage reduction.

A rundown situation causing voltage instability occurs when load dynamics attempts to restore power consumption beyond the capability of the transmission system and the connected generation [1], while the most common form of voltage instability is the progressive drop in bus voltages, the possibility of overvoltage instability also exists and has been experienced at least on one system [2]. It can occur when EHV transmission lines are loaded significantly below surge impedance loading and under excitation limiters prevent generators and/or synchronous condensers from absorbing the excess reactive power. Under such conditions, transformer tap changers, in their attempt to control load voltage, may cause voltage instability. Voltage stability problems may also be experienced at the terminals of HVDC links. They are usually associated with HVDC links connected to weak AC systems. The HVDC link control strategies have a very significant influence on such problems. Low voltage profile may results into the voltage collapse of the system which is responsible for causing blackouts. Therefore, it becomes essential to analyze the voltage stability. Main causes for the voltage instability, are listed below [1]:

1. Increased loading of transmission lines.

2. Reactive power constraints.

3. Load characteristic at low voltages.

4. Response of On Load Tap Changing (OLTC) transformer.

5. Unexpected relay operations.

The voltage stability has been further classified into four categories: Large disturbance voltage stability, small disturbance voltage stability, short term voltage satiability and long term voltage stability. A summary of these classifications is as follows [2]:

A. Large disturbance voltage stability: It refers to the system's ability to maintain steady voltage following large disturbances such as, system faults, loss of generation or circuit contingencies. This ability is determined by the system load characteristics and interaction of both continuous and discrete controls and protections. The study period of interest may be from few seconds to tens of minutes. This requires long term dynamic simulation study of the system to capture the interactions of under-load tap changer and generator field current limiter. If following a large disturbance and subsequent system control actions, voltages at all the buses in the system settle down at acceptable levels, the system is said to be large disturbance voltage stable.

B. Small-disturbance voltage stability: This stability is concerned with the ability of the system to maintain acceptable level of steady voltages, when subjected to small perturbations such as incremental changes in system load. This form of stability is also influenced by the characteristics of loads, continuous controls, and discrete controls at a given instant of time. The basic processes contributing to small disturbance stability are essentially of a steady state nature. Therefore, static analysis can be effectively used to estimate stability margins. 
C. Short term voltage satiability: It involves dynamics of fast acting load components such as induction motors, electronically controlled loads and HVDC converters. The study period of interest is in the order of several seconds and the analysis requires solution of appropriate system differential equations.

D. Long term voltage stability: The study of long term voltage stability involves the dynamics of slower acting equipment such as tap changing transformers, thermostatically controlled loads and generator current limiters. The study period of interest may extend to several or many minutes, and requires long term dynamics system simulation.

Some counter measures to prevent voltage collapse are [1]:

- Switching of shunt capacitors

- Blocking of tap-changing transformers

- Re-dispatch of generation

- Load shedding

- Temporary reactive power overloading of generators

\section{DIFFERENT TOOLS AND METHODS FOR ANALYZING VOLTAGE STABILITY}

Some of the tools used for the analysis of stability voltage are the methods based on Static and dynamic analysis.

A. Static analysis: They consist in the solution of the set of algebraic equations that represent the system in steady state [1], with the aim of evaluating the feasibility of the equilibrium point represented by the operating conditions of the system and to find the critical voltage value. The advantage with respect to the dynamic analysis techniques is that it gives valuable information about the nature of the problem and helps to identify the key factors for the instability problem. The plotting of the PV curve helps to the analysis of the voltage stability limits of a power system under a scenario with load increments and with the presence of a disturbance such as the loss of generation or the loss of a transmission line.

B. Dynamic Analysis: They consist in the numerical solution of the set of differential and algebraic equations that model the power system [1], this is similar as transients; however, this kind of simulations need considerable amount of computing resources and hence the solution time is large and they do not give information about the sensibility and stability degree.

C. PV curves:The PV curves represent the voltage variation with respect to the variation of load reactive power [1]. This curve is produced by a series of load flow solutions for different load levels uniformly distributed, by keeping constant the power factor. The generated active power is proportionally incremented to the generator rating or to the participating factors which are defined by the user. The $\mathrm{P}$ and $\mathrm{Q}$ components of each load can or cannot be dependant of the bus voltage accordingly to the load model selected. The determination of the critical point for a given load increment is very important because it can lead to the voltage collapse of the system. These characteristics are illustrated in Figure (1).

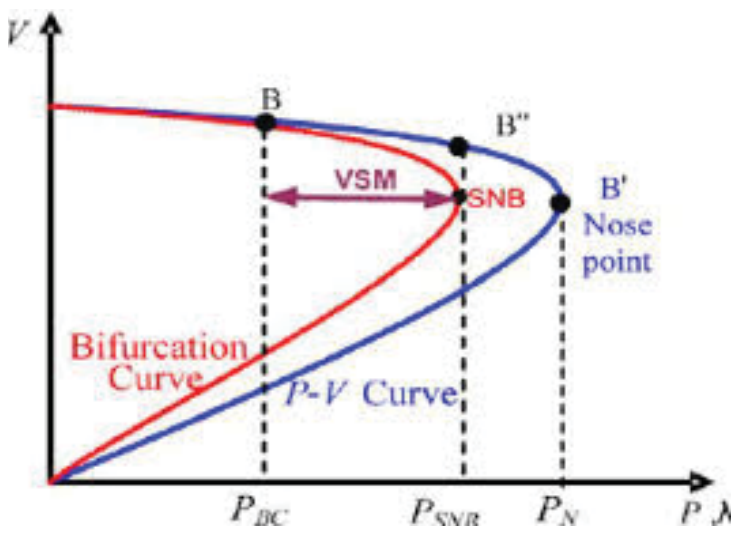

Figure (1): PV curve 
D. CONTINUATION POWER FLOW METHOD: The continuous load flow [3] [4] procedure is based in a reformulation of the equations of the load flow problem and the application of the continuation technique with a local parameterization which has shown to be efficient in the trajectory plotting of PV curves. The purpose of continuous load flows is to find a set of load flow solutions in a scenario where the load is continuously changing, starting from a base case until the critical point. Thereafter, the continuous load flows had been applied to understand and evaluate the problem of voltage stability and those areas that are likely to the voltage collapse. Besides, they have also been applied in other related problems like the evaluation of power transfer limits between regions.

E. OPTIMAL POWER FLOW: An indicator termed as L -indicator which is derived from Kirchhoff law to determine voltage instability in [5]. This voltage stability indicator predicts the voltage stability margin of current operating point. The value of $\mathrm{L}$-indicator lies in between 0 and 1 . Lower the value of $\mathrm{L}$ - indicator greater is the stability margin. By using L-indicator it is used to find the impact of loads, area and power transaction. Optimal power flow by using Newton-Raphson method can be simulated using any Power System Software. Indicator predicts the voltage stability problem accurately and properly Load curtailment is one of the methods for voltage stability margin criteria of the power system [6]. For the computation of load curtailment an optimal power flow along with steady state voltage stability indicator is used.

F. PARTICIPATION FACTOR FOR MW AND MVAR MANAGEMENT: Carolina [7] suggested a methodology to improve the voltage stability margin by economical dispatch. It is based on the methodology of active or reactive power re-dispatch for working of normal condition and also minimum load shedding during contingencies. It is to be determined by modal participation factor which is having impact on the voltage system margin and load shedding. Participation factor is obtained from the critical Eigen vector of Jacobian matrix. By using participation factor the critical area is easily found. Participation factor is obtained by optimal power flow. Modal participation factor indicates which generator should supply more or less active or reactive power for the voltage stability margin. This information is then included in the system dispatch problem and leads to final decision. Participation factor is also used for identifying most adequate buses in the system for the purpose of load scheduling. Taciana [8] proposes a methodology based on optimizing generator and synchronous condenser of reactive power injections in the system. Participation factor based on the optimal power flow is calculated which aims to provide the adequate reactive power to the system and prevent the instability of the system. Reactive power reserve management is a method for voltage stability margin, based on the optimal power flow [9]. Participation factor for each generator is calculated on the basis of Q-V curve methodology. The Bender's decomposition methodology is applied to the reactive reserve management problem. The reactive reserve management program based on the optimal power flow is used to manage the critical reactive power reserves. Complete analysis is done for the reactive power management for improving the voltage stability of the system. Both static and dynamic voltage stability is improved by this method.

G. VOLTAGE STABILITY INDICES: Some authors have proposed voltage stability indexes which are based in some kind of analysis of load flows with the aim to evaluate the stability voltage limits. However, the Jacobian used in the load flows, when the Newton- Raphson is employed, becomes singular at the critical point, besides the load flow solutions at the points near to the critical region tend to diverge [1]. Voltage stability analysis is done on the basis of various indices [10]. This paper performs the comparison of various stability indices for the analysis of voltage stability system on IEEE 14 bus system. Analysis of this method is done by using indices like P-V curve and Q-V curve, L-index, V/V0 index, modal analysis, line stability index Lmn, line stability index FVSI, line stability index LQP, line stability index VCPI are used for the analysis. It shows the comparative study along with the analysis of performance of some online static voltage collapse indices. Estimating the load ability of the power system is very important and it is done by using voltage stability indices (VSI) [11]. It gives the distance from current operating point to collapse point. The comparisons and effectiveness of this method is studied on IEEE 14 bus system. The analysis of this method gives two parts: (1) Proximity of voltage instability (2) Weak bus and area in the system. The main purpose of these indices is to find out the point of voltage instability and weak bus in the system. The analysis and study of system is done by using line VSI and nodal VSI for detecting the weakest area and load ability of the system. Indices provide correct predictions which provide an alarm signal for the prevention from instability.Four indices are used for the voltage stability in transmission line and system buses in [12]. Two indices are used for the system buses termed as VPI bus and VQI bus for the study of dynamics of load and generator. The remaining two indices 
VPI line and VQI line are used for transmission line voltage stability for the study of transmission stress and outage. These indices are fast, accurate and provide the information about maximum active and reactive power transfer in the system. This analysis is done on the IEEE 14 bus system and IEEE 118 bus system. The analytical result obtained from the indices, helps to avoid voltage collapse and prevent the system from voltage instability.

H. VOLTAGE STABILITY INDEX METHOD: D. Thukaram [13] proposes the analysis of steady state voltage stability. It is based on new operational load flow and sensitivity of reactive control variable. It mainly deals with the analysis and enhancement of steady state voltage stability based on the L index method Tellegen's theorem and adjoin network derive the new voltage stability index in [14]. This index provides different ways for curve fitting from two consecutive phasor measurements. The main purpose for the development of local method is that local phasor directly gives the voltage instability. New online voltage stability index based on synchronized phasor measurement is proposed to find the voltage stability of the system [15]. This index is mainly designed for the determination of maximum transferable power. For the application of this index to large power system it is converted into single source power system. The voltage stability index is applied on the two power system. If the value of the index tends to zero it represents the voltage collapse condition. The output obtained from the voltage stability indices is simple, accurate and reliable hence useful for the online application of the system.

I. SYNCHROPHASOR METHOD: Early detection of voltage instability in the system is always preferable than the actual occurrence voltage instability state. The area detection of voltage instability is obtained from the synchronized phasor measurement method [16]. Initially, set of algebraic equations are solved and sensitivity is found for the identification of combination of load powers. The computation of voltage instability is found by the sensitivity of the system before low voltage occurs and the effect of noise is also taken into consideration. The effectiveness of the system is observed based on availability of Phasor Measurement Unit (PMU) configuration of the system to give the exact status of voltage instability. Finally impedance matching criteria is provided but it shows poor significance in case of large-disturbance scenario. It gives the early detection of impending voltage instability. Heng-Yi Su [17] proposes an approach that secondary voltage control using synchronized phasor measurement as a control feedback in power system. The main purpose is to improve the voltage stability by proper management of reactive power against diverse load condition. This method is feasible where load disturbances are estimated on-line for computing and feasible control input for minimization of deviation of voltage under load variable condition. Main concern is on the secondary side for maintaining the constant voltage by proper adjustment of reactive power. A new adaptive synchrophasor based SVC scheme is proposed having the application of PMU with time as a control signal.

J. APPLICATIONS OF AI TOOLS AND EVOLUTIONARY ALGORITHMS: For Voltage Stability analysis AI tools like Artificial Neural Networks and Fuzzy Logic and Evolutionary Algorithms like Genetic Algorithm, Ant Colony Algorithm, Differential Evolution Algorithm, Bacterial Foraging Optimization Algorithm etc can be used for simulation using any Software.M. Sedighizadeh [18] proposes optimal distributed generation allocation for the improvement in voltage stability and reduction of loss in the transmission line. The tool used for this method is genetic algorithm. Distributed generation helps in the improvement of voltage stability profile. MATPOWER package is used for load flow and it is analyzed in MATLAB software along with ETAP for correctness of the system. The Khoda Bande Loo distribution test feeder in Tehran has been evaluated for voltage profile improvement by application genetic algorithm. Superconducting magnetic energy storage (SMES) may help to improve the stability of power system. SMES consist of isolated transformer, voltage source convertor (VSC), DC convertor (DC/DC) and a superconducting magnetic coil [19]. It uses voltage stability index and genetic algorithm method for optimization of SMES location. Artificial neural network is used for online voltage stability of the system in [20]. In this system, the most vulnerable load bus system is determined by the modal analysis method. For the vulnerable load bus system a separate feed forward type of ANN is trained. The ANN's are trained for the different loading condition of the system, which computes the active and reactive power of the system. Analysis of voltage stability in the multi-bus power system is done by using artificial neural network and linear voltage stability indicator in [21]. Linear voltage stability indicator is used for the prediction of voltage collapse of the system while its combination with the ANN gives an estimation of voltage security of the system. ANN is used for the online monitoring of voltage stability of the system. S. Senthil Kumar [22] deals with the estimation of steady state of voltage stability. The analysis is done 
on the basis of L-index method modeled in terms of fuzzy set by using triangular membership function. This method gives proximity of voltage collapse before system become unstable.

K. BIFURCATION ANALYSIS: Bifurcation theory assumes that system parameters vary slowly and predicts how the system typically becomes unstable. The main idea is to study the system at the threshold of instability. Regardless of the size or complexity of the system model, there are only a few ways in which it can typically become unstable and bifurcation theory describes these ways and associated calculations. Many of these ideas and calculations can be used or adapted for engineering purposes. A saddle-node bifurcation point of the power flow equations of a power system can provide information regarding the margin of static voltage stability at the current operating point of the power system. The information is used in voltage stability control. Over the last few years, we have seen several methods for computing saddle-node bifurcation points of power flow equations. For example, the continuation' methods and the direct methods have been used extensively to compute saddle-node bifurcation points. While these methods provide general-purpose algorithms for computing saddle-node bifurcation points of power flow equations, the efficiency of these methods can be greatly improved if the special structures of the power system models are taken into account. Ian Dobson [23] gives a method for calculation of closet saddle node bifurcation point of the system. New iterative and direct method is used for the calculation of load power at which bifurcation occurs. The nature of the load cannot be estimated hence worst case load power margin is found out for the prevention from voltage collapse. It also gives the direction of load flow when the load increases. Jin $\mathrm{Lu}$ [24] proposes two methods for computing the saddle node bifurcation point. First method computes the saddle node bifurcation point along a given ray of power flow equation in the parameter space that can be calculated by optimization technique. Another method used for the computation of saddle node bifurcation point is locating closet saddle node bifurcation point with the current operating point. This method uses an iterative approach for the computation. The fast computation method is proposed to estimate the load power margin with respect to saddle node bifurcation point under the generator and branch contingencies for the evaluation of voltage stability in [25]. The method proposed is based on the linear sensitivity. Linear estimations are taken into account by considering the non linearities of power flow equations and fast search technique. Magnus Perninge [26] proposed the risk estimation of critical time to voltage instability by saddle node-bifurcation with various loading condition. Stochastic method is used for predicting the voltage collapse. For the estimation of accuracy of the system Monte Carlo Simulation method is used. The difference between actual critical time and estimated critical time depends on the distance from current operating point to saddle-node bifurcation set. The uncertainties are modeled by linear combination with time which is changed by Brownian motions. The method employed is fast and reliable and can be used by daily operations.

L. CHAOTIC THEORY: Chaotic behavior in power systems has been studied in relatively simple and theoretical system models, where some particular assumptions are made to represent the system as a set of ordinary differential equations (ODE), using "special" nonlinear system analysis tools. Chaotic phenomena in relatively simple power system models has been observed and studied for the past two decades. For example, in [27]-[29], chaotic behavior of a simple 3-bus power system is studied in detail, where the single system load is represented with a dynamic model to characterize the system using ordinary differential equations (ODE). In [30], on the other hand, a larger 9-bus test system is studied using an ODE model (with dynamic loads), illustrating the interactions of chaotic motions and system dynamic components; transmission system controllers are then used to prevent or eliminate chaotic oscillations. The study of chaotic behavior in nonlinear systems is typically carried out using a combination of analyses techniques [31].

M. TOOLS AVAILABLE FOR VOLTAGE STABILITY ANALYSIS: This paper presents a MATLAB based Voltage Stability Toolbox (VST) [32] designed to analyze bifurcation and voltage stability problems in electric power systems. VST combines proven computational and analytical capabilities of bifurcation theory, and symbolic implementation and graphical representation capabilities of MATLAB and its toolboxes. The motivation for developing the package is to provide a flexible simulation environment for an ongoing research conducted at the Center for Electric Power Engineering (CEPE) of Drexel University, Philadelphia, PA, and to enhance undergraduate/graduate power engineering courses. VST is a very flexible tool for load flow, smallsignal and transient stability, and bifurcation analysis. Some of the tools available in the market for analysis of Voltage Stability are PSAT, VSAT, MATEMTP, PAT, EST, SPS and MATPOWER.

\section{CONCLUSIONS}


Voltage stability plays a very key role in Power System Operation and Control and also in maintaining the security of the Power System. Various causes for Voltage Instability and preventive measures are elucidated in this paper. To analyze Voltage Stability, different methods available in the market are Optimal Power Flow, Continuation Power Flow, Voltage Stability Indices Calculation Method, Bifurcation Analysis and finding Chaotic Conditions. A detailed survey on Voltage Stability and different techniques available in the literature are explained in this paper. In this paper, Toolboxes available in the literature for analyzing Voltage Stability are also presented.

\section{REFERENCES}

[1] A Text Book by Thierry van Cutsem and Costas Vournas, "Voltage Stability of Electric Power Systems", Springer US, 1998.

[2] A Text Book by P Kundur, "Power System Stability and Control", Tata McGraw Hill Company Private Limited, 2008.

[3] Parul Anand, U., Dharmeshkumar, P., "Voltage Stability Assessment Using Continuation Power Flow", International Journal of Advanced Research in Electrical, Electronics and Instrumentation Engineering, Vol. 2, Issue 8, August 2013.

[4] Arthit Sode-Yome, Mithulananthan, N., "Static Voltage Stability Study Using MATLAB Symbolic and Optimization Toolboxes", Royal Thai Government and Siam University, Thailand, January 2006.

[5] Kumaraswamy, Jahnavi, W.V., Devaraju, T., Ramesh, P.,"An Optimal Power Flow (OPF) Method with Improved Voltage Stability Analysis", Proceedings of the World Congress on Engineering London, U.K. Vol. II, July 4 - 6, 2012.

[6] Garng Huang, M., Nirmal-Kumar, C., "Voltage Stability Constrained Load Curtailment Procedure to Evaluate Power System Reliability Measures", IEEE, power engineering society winter meeting, Vol.2, 2012, pp: 03-07.

[7] Carolina Affonso, M., Luiz da Silva, C.P., Flavio Lima, G. M., Secundino Soares, "MW and MVAR Management on Supply and Demand Side for Meeting Voltage Stability Margin Criteria", IEEE Transactions on Power Systems, Vol. 19, no. 3, pp: 1538-1545, August 2004.

[8] Taciana Menezes, Lulz da Silva, C.P., Carolina Affonso, Vivaldo da Costa, F., Secundino Soares, “ MVAR Management on the Predispatch Problem for Improving Voltage Stability Margin", IEEE Proceedings Generation, Transmission and Distribution, Volume no.151, no. 6, pp: 665-672, February 2002.

[9] Feng Dong, Badrul Chowdhury, H., Mariesa Crow, L., Levent Acar, "Improving Voltage Stability by Reactive Power Reserve Management", IEEE Transactions on Power Systems, Vol. 20, no.A1, pp: 338-345, February 2005.

[10] Claudia Reis, Maciel Barbosa, F.P., "A Comparison of Voltage Stability Indices",Electrotechnical Conference, Melecon, Benalmadena (Malaga), Spain, pp: 1007-1010, May 16-19, 2006.

[11] Suganyadevi, M.V., Babulal, C.K.., "Estimating of Loadability Margin of a Power System by Comparing Voltage Stability Indices", International Conference on Control, Automation, Communication and Energy Conservation 2009, pp: 01-05, 4th-6th June, 2009.

[12] Althowibi, F.A., Mustafa, M.W., "Power System Voltage Stability: Indications, Allocations and Voltage Collapse Predictions", International Journal of Advanced Research in Electrical, Electronics and Instrumentation Engineering, Vol. 2, Issue 7, July 2013.

[13] Thukaram, D., Parthasarathy, K., Khincha, H.P., Narendranath Udupa, Bansilal, A., "Voltage stability improvement: Case Studies of Indian Power Networks", Electric Power Systems Research, Vol.44, no 1, pp: 35-44, India.

[14] Ivan Smon, Gregor Verbic,, Ferdinand Gubina, "Local Voltage-Stability Index Using Tellegen's Theorem", IEEE Transactions on Power Systems Vol. 21, no. 3, pp: 1267-1275, August 2006.

[15] Yanfeng Gong, Noel Schulz, Armando Guzmán, "Synchrophasor-Based Real-Time Voltage Stability Index", Power Systems Conference and Exposition, pp: 01-08, December 2006.

[16] Mevludin Glavic, Thierry Van Cutsem, "Wide-Area Detection of Voltage Instability from Synchronized Phasor Measurements Part II: Simulation Results", IEEE Transactions on Power Systems, vol. 24, no. 3, pp: 1417-1425, August 2009.

[17] Heng-Yi Su, Chih-Wen Liu, “An Adaptive PMU-Based Secondary Voltage Control Scheme”, IEEE Transactions on Smart Grid, Vol. 4, no. 3,pp: 1514-1522, September 2013.

[18] Sedighizadeh, M., Rezazadeh, A., "Using Genetic Algorithm for Distributed Generation Allocation to Reduce Losses and Improve Voltage Profile", World Academy of Science, Engineering and Technology, Vol. 37, 2008, pp: 251-256.

[19] Xiaohua Huang, Guomin Zhang, Liye Xiao, "Optimal Location of SMES for Improving Power System Voltage Stability", IEEE Transactions on Applied Superconductivity, Vol. 20, no. 3, pp: 1316-1319, June 2010.

[20] BhavikSuthar, Balasubramanian,R., "A Novel ANN Based Method for Online Voltage Stability Assessment", 14th International Conference on Intelligent System Applications to Power Systems, ISAP Kaohsiung, Taiwan, November 4 - 8, 2007.

[21] Kabir, Chakraborty, Abhinandan De, Abhijit Chakrabarty, "Assessment of Voltage Security in Multi- Bus Power System Using Artificial Neural Network and Voltage Stability Indicator", Journal of Electrical System 6-4, pp: 517-529, 2010.

[22] Senthil Kumar, S., Ajay Vimal Raj, P.,"Fuzzy Logic based Stability Index Power System Voltage Stability Enhancement”, International Journal of Computer and Electrical Engineering, Vol. 2, no. 1, February, 2010.

[23] Ian Dobson, Liming Lu, "New Methods For Computing A Closet Saddle Node Bifurcation And Worst Case Load Power Margin For Voltage Collapse", IEEE transaction on Power System, Vol. 8, no-3, pp: 905-913, August 1993.

[24] Jin Lu, Chih-Wen Liu, James S. Thorp, "New Methods for Computing a Saddle- Node Bifurcation Point for Voltage Stability Analysis", IEEE Transactions on Power Systems, Vol. 10, no. 2, pp: 978-989, May 1995.

[25] Yorino,N. H., Li, H. Q., Harada, S., Ohta, A., Sasaki, H., "A Method of Voltage Stability Evaluation for Branch and Generator Outage Contingencies", IEEE Transactions on Power Systems Vol. 19, no. 1, pp: 252-259, February 2004.

[26] Magnus Perninge, Valerijs Knazkins, Mikael Amelin, Lennart Söder, "Risk Estimation of Critical Time to Voltage Instability Induced by Saddle-Node Bifurcation”, IEEE Transactions on Power Systems, Vol. 25, no. 3, pp: 1600-1610, August 2010.

[27] V. Ajjarapu and B. Lee, "Bifurcations Theory and its Application to Nonlinear Dynamical Phenomena in an Electrical Power System," IEEE Trans. Power Systems, vol. 17, no. 1, pp. 424-431, 1992.

[28] H.-D. Chiang, C.-W. Liu, P. P. Varaiya, F. F. Wu, and M. G. Lauby. "Chaos in a Simple Power System," IEEE Trans. Power Systems, vol. 8, no. 4, pp. 1407-1417, Nov. 1993.

[29] H. O. Wang, E. H. Abed, and A. M. A. Hamdan, "Bifurcations, Chaos, and Crises in Voltage Collapse of a Model Power System," IEEE Trans. Circuits \& Syst. I, vol. 41, no. 3, pp. 294-302, Mar. 1994.

[30] K. N. Srivastava and S. C. Srivastava, "Elimination of dynamic bifurcation and chaos in power systems using FACTS devices," IEEETrans. Circuits \& Syst. I, vol. 45, no. 1, pp. 72-78, 1998. 
[31] R. Seydel, Practical Bifurcation and Stability Analysis: From Equilibrium to Chaos, Springerr-Verlag, 1994.

[32] Saffet Ayasun,Chika O. Nwankpa and Harry G. Kwatny, "IEEE Voltage Stability Toolbox for Power System Education and Research", IEEE transactions on Education, Vol. 49, No. 4, pp 432-442,November 2006 